\title{
Asset Supply Chain Management System-based IoT Technology for Higher Education Institutions
}

\author{
https://doi.org/10.3991/ijoe.v15i03.8533 \\ Watjanarat Kuandee( $\left.{ }^{\varpi}\right)$ \\ Surindra Rajabhat University, Surin, Thailand \\ watjanarat.kesrru.ac.th \\ Prachyanun Nilsook, Panita Wannapiroon \\ King Mongkut's University of Technology, Bangkok, Thailand
}

\begin{abstract}
The purpose of this research is to design and develop an asset supply chain management system-based IoT technology for Higher Education Institutions (HEIs). Although nowadays there are computer-based asset information management systems, the problem is that such a system cannot immediately monitor the status of the asset. There is some information that is not related to reality, including a lack of asset movement records and information as to the status of each asset, which could lead to the mistake in terms of the working process which is unable to control and monitor a situation. Therefore, this research applied the concept of supply chain management (SCM) and IoT technology in order to unsure good governance on the part of HEIs. This research is focused on durable articles and the use of a Near Field Communication (NFC) tag to communicate and exchange information with NFC-compatible smartphones. In this case, it can identify and monitor the asset under consideration and also assess associated information from any device. This solution can increase efficiency and value in terms of the organization. Moreover, the design diagram of the system leads to the development of asset supply chain management system (ASCMS)-based IoT technology for HEIs. The evaluation of the performance of this technology shows that the system operates at a great level and that ASCMS-based IoT technology for HEIs it can be applied to ensure the good governance of such institutions. In addition, this research can serve the internal organizational administration of Thai HEIs, involving dealing with working processes in line with real-life situations, relating to budget management, organizational working behaviors, and service users' requirements. All of these aspects are analyzed based on good governance, including the rules of law, transparency and values.
\end{abstract}

Keywords - Asset Supply Chain Management, Internet of Things, Near File Commination 


\section{Introduction}

Global changes which have emerged in both digital and physical worlds result from new technological phenomena that lead to people's final decision-making with regard to their business, marketing and career activities in different organizations [52]. In fact, technology is integrated into everything, resulting in a digital service based on intelligent systems. Stepping into the digital world, HEIs need to be driven by advanced information technology to streamline workflows [2][3]. Not only does technological innovation have an important role to play in HEIs, but it also focuses on innovative strategies and information technology designed for dealing with new innovations which promote added value within HEIs. Moreover, the number of HEIs' assets used for different management purposes can link to asset control and monitoring.

Changing storage locations, together with a lack of updated databases and a failure to use valuable assets are induced by monitoring such a different asset items spent for its longer time. Even if computers are used to store data, it may take several days to track an asset in such a way as to get the information that is consistent with a real-life situation. Therefore, mistakes in terms of ASCMS mostly occur during the working process.

In order to enhance accurate asset flows appropriate for an HEI's circumstances, as well as to offer data security, low power use, and user-friendliness [7], this study conceptualizes that both IoT technology and SCM through the use of the android operating system, mobile applications and web applications, can be used in order to identify and investigate a required feature of HEIs. Moreover, correct user authentication and authorization is essential when it comes to log-in, because its cloud computing application can be accessed anywhere and anytime from any device, allowing a user to easily control and monitor assets.

\section{$2 \quad$ Related Work}

\subsection{Asset supply chain management}

HEIs' assets relate to a range of university activities, so that effective asset management is needed to benefit the organization [47][48][53]. Therefore, the concept of SCM is used to develop the ASCMS in order to effectively manage related activities, and enhance the relationship of existing assets within an organization. The ASCMS process is divided into four main processes: asset registration, asset control and maintenance, monitoring asset management, and asset distribution [39].

Logistics relationships within the supply chain are divided into five parts: (1) Information flows emphasizing the importance of exchanging accurate real-time information between units; (2) Financial flows and payments for suppliers in the form of debits and credits; (3) Asset flows along the chain, reverse flows in terms of returned products, as well as office supplies under maintenance and destroyed office supplies; (4) Demand in the form of the number of effective asset demands at reasonable prices 
for each university campus, and (5) Supply in the form of assets taken from required purchasing management [15][39]. Thus, changes that occur in terms of quantities demanded and quantities supplied within the supply chain directly affect not only the equilibrium price, but also the equilibrium quantity. Therefore, managing the flows of things that occur in each step of the supply chain is an important part of the supply chain, with regard to the added value, the value chains and the successes of Thai HEIs [15][50].

The function of ASCMS involves information-sharing within the system and between systems connected through its IoT Technology, which leads to effective, wellorganized management, and the control of assets [9]. Furthermore, devices display information immediately when smartphones are near, due to the NFC tags that are attached to each device within its specified period. As a result, asset monitoring is done quickly, and the information noted above are also shared as part of an effective management process [15][42][44][50]. The applications of SCM and ASCMS are based on the needs of Chief Executive Officers (CEO), assets officers, and asset management officers dealing with asset-purchasing on the university campus. In terms of good governance related to the rules of law, transparency and value, the application of ASCMS in HEIs has led to well-organized organizational administration [23][53].

\subsection{The Internet of Things (IoT)}

IoT refers to the internet technology that is used to connect devices and physical objects [6][56]. The RFID group defined the IoT as a global network of interconnected objects which are addressed through a standard communication protocol [11][24][40]. For example, mobile phones are used to control or instruct devices to work at nearer and longer distances by linking directly or communicating via the Internet, respectively. It can also control systems such as turning them on and off, switching on air conditioners, etc., from anywhere in the world. In fact, technological applications all employ different devices and accessories for convenience [14][16][55].

Most importantly, IoT technology is based on the concept of Radio Frequency Identification Tags (RFID Tags) so that all objects leading to the specific location of any device can be identified and possibly located around the world [22]. In addition, storing information can occur in interchanging devices [56], and all smart objects can be connected [31]. Thus, a world-wide network of interconnected objects is uniquely addressable, based on standard communication protocols [8][34][35][51]. That is to say, there are many different types of devices involved in this process. However, this concept has transformed itself into a vision that connects the real world and the digital world, and the concept of IoT has brought about the change in ICT innovation that we see today.

In Figure 1, key concepts, technologies, and indicators classified in terms of the vision of IoT can be sorted into three major categories: internet-oriented (middleware); things-oriented (sensors), and semantic-oriented (knowledge) [6][24]. 


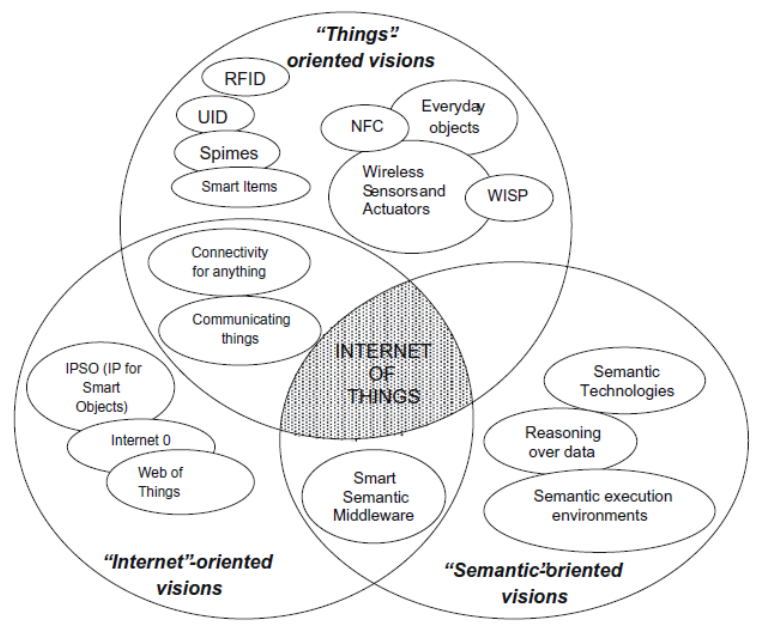

Fig. 1. "Internet of Things" paradigm as a result of the convergence of different visions $[6][24]$.

From this figure, we can see that the IoT technology has resulted in the convergence of three visions, referred to as "the things-oriented vision." This means that "Things" such as RFID, NFC, etc. are mainly utilized in order to act as basic devices [41][54]. Moreover, those devices are used for searching for objects, statuses, and current location, and the use of RFID is supported by the Auto-ID Labs in research collaboration with the EPC global [5].

With regard to the internet-oriented vision, devices can be connected anywhere [6][21][38][43]. In addition, the IoT technology is not only implemented by the internet protocol (IP), but it is also used as a technological network connected with intelligent devices around the world, in order to control battery-operated embedded devices [19][21][32], as well as to access such things or devices anywhere [21]. As a result, things or devices can automatically communicate with other computers and services, so that all of them can be used to facilitate human beings' social interaction [19]. Changes caused by the internet will decrease in the digital world, whereas its speed results from the connection of objects used in everyday life. Thus, IoT technology has become a device designed for developing interconnected applications and services, with working collaborations being mostly found at a high level.

According to the semantic-oriented vision with emphasis on fundamental ideas, many devices will be used in communicating and connecting to the internet. Therefore, data collection, storage, replacement, searching, as well as interconnection designed with the concept of IoT technology, are extremely challenging [12][33][38][58].

Semantic technologies will also play a significant role in IoT in relation to semantic execution environments, IoT-oriented architectures, as well as storage flexibility in order to meet the requirements of communication infrastructure [1][10][18][58]. Moreover, web standards are being used once more to connect and integrate with the 
Web of Things, which are merged into the part of the real world shown in the World Wide Web (WWW), and are simplified for IoT applications [25][26][27][28][29][30]. The relevant web standards are as follows:

- The Social Web: Open services and web engagement are designed to create and support virtual communities such as OAuth, OpenID, Social Networks, etc. within social networks [57];

- The Semantic Web: Contents are embedded in readable computers, and data are interpreted within websites. In addition, more captions are illustrated for insightful descriptions, and data are parsed from metadata. As it is indexed by search engines, tools and techniques in data dissemination such as JSON-LD, Micro-formats, etc. are published, connected, searched, and created on the web [38];

- The Real-Time Web: Many domains rely on real-time data to deliver outcomes in a timely manner, and on-line services on information technology such as WebSockets, etc. are needed for quick delivery [57];

- The Programmable Web: The ability to access data and APIs-opened services such as REST, HTTP, JSON, etc., allows developers to easily create new mashups [57], and

- The Physical Web: This extends to access in terms of information technology to websites connected to physical objects, GPS sensor-installed smartphones, internet connections, applications identified for different locations, Foursquare-converted Google Place services designed for creating the Physical Web [57]. Its delivered contents depend on its physical location.

\subsection{Near field communication (NFC)}

NFC is a wireless technology relating to close distance frequency. It is controlled by Radio-Frequency Identification (RFID), which activates a contactless system operating at a distance of between 4 and $10 \mathrm{~mm}$ [9]. It is also a wireless communications technology that brings the principle of RFID technology into shaping human social interaction [13][17][20]. Using tags or smartcards, as well as identifying read-to-use devices, can be also done using its NFC characteristics in order to automatically access the system without resetting [45][46][49]. In terms of the advantages of NFC, users' personal security, low use of power, and user-friendliness are mostly satisfied due to its operational short range, which is less vulnerable to data-spying than other wireless technologies [7][9]. Most importantly, one thing that makes NFC stand out is its automatic speed of performance with regard to matching devices. Contactless tags can be used for various tasks such as e-finance, e-banking, e-ticket, warehouse management, etc.

NFC and RFID real-time processing technologies can track and monitor real-time movement that happens in all SCM formats. Designing involving modern packaging, raw material purchasing, production, transport database management, distribution of products and semi-products, as well as product-refunding, and after-sales services can also be monitored in this way [6][15]. 
In addition, data services related to manufactured products are taken in terms of organizations' supply chains responding to marketing complexities. The results of integrated application show that 120 days are used as the response time of traditional organizations in terms of dealing with their customers' requirements with regard to product purchasing. On the other hand, the IoT applications of leading companies such as Wal-mart and Metro take only a few days, because the IoT system can not only check products in stock, but their security can also be facilitated [59].

However, NFC, wireless sensors and actuator networks (WSAN), as well as RFID are all accepted as key components that can be connected to the real world and the digital world [4][6][12]. In addition, devices such as those offering machine to machine communication (M2M), etc. will be able to be connected to the internet in the future $[22][43]$.

\section{System Analysis and Design}

\subsection{Stakeholders}

The design of the ASCMS are used by two major groups that include:

- Administrators who are responsible for planning, controlling, and managing the ASCMS in terms of users' databases; moreover, regulations as to its web applications, authorization, and users' service cancellation procedures are also set up;

- Users are a group whose administrator's privileges are offered in terms of internal data accessibility, depending on the group's various privileges.

The users' duties and job descriptions are divided into three major categories as follows:

- The Chief Executive Officer (CEO) is an HEI executive who can access all data within the HEI system. The CEO's asset reports can also be implemented for final decisions on effective strategic management with regard to various aspects;

- Asset officers are those who manage all available assets through a web application. Their main tasks include asset registration, NFC recording, controlling and maintenance of assets, as well as sales distribution;

- Asset management officers are those who perform their monitoring asset management, and update their asset information through the mobile applications associated with the system.

\subsection{Process diagram}

The IoT ASCMS process diagram illustrates a process by which smart phones and assets can communicate automatically with a sensor. Furthermore, NFC is a communication medium over the Internet that is used to identify each asset's data. The IoT process is divided into two major aspects as shown in Figure 2.

- Monitoring asset management: The asset management officers use an NFCcompatible smartphone in order to send a radio signal to detect assets in the area. 
When the system is connected with the asset's Tag ID, it will show the information on the smartphone with regard to the asset. If the information does not match with the reality, the officers can save and edit the information real-time. The information will automatically be saved and stored on the central system. The technology behind the system will request/respond to the information through TCP/IP on the internet $(3 \mathrm{G}, 4 \mathrm{G}, \mathrm{WiFi})$ by exchanging the information in the JSON format through the HTTP protocol to the REST service interface on the central system to GET/POST information, and will process and store it on the web server and the database server using cloud computing. The central system uses web service technology which is part of the representational state transfer service (REST Service).

- Data management: Asset officers manage their equipment using a computer via the ASCMS web application, and data about assets can be added, deleted, modified, and updated. Also, reported assets can be reviewed in the system.

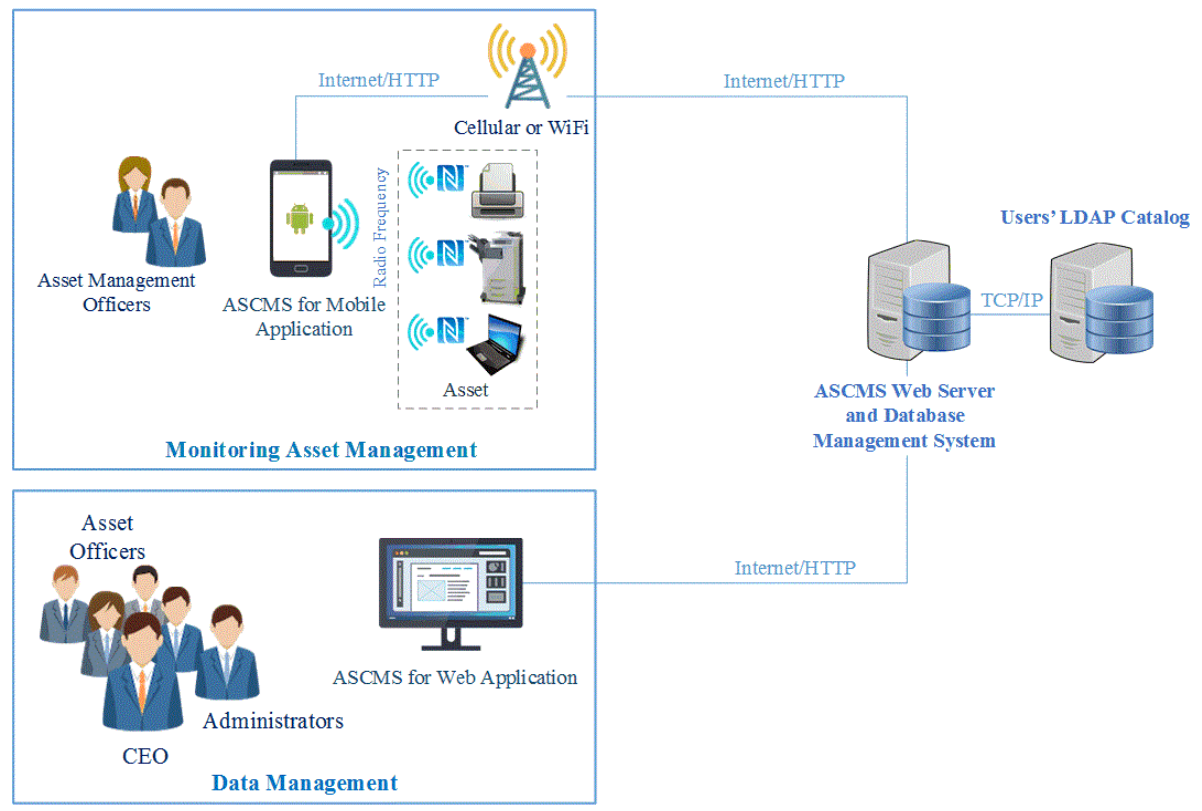

Fig. 2. IoT Process Diagram of ASCMS

\subsection{Sequence diagram designed for monitoring assets}

In terms of designing the ASCMS-based asset model found in the sequence diagram, it is designed as a dynamic model or behavioral model that encourages internal activities found in the ASCMS, which interacts with another object as shown in Figure 3. 


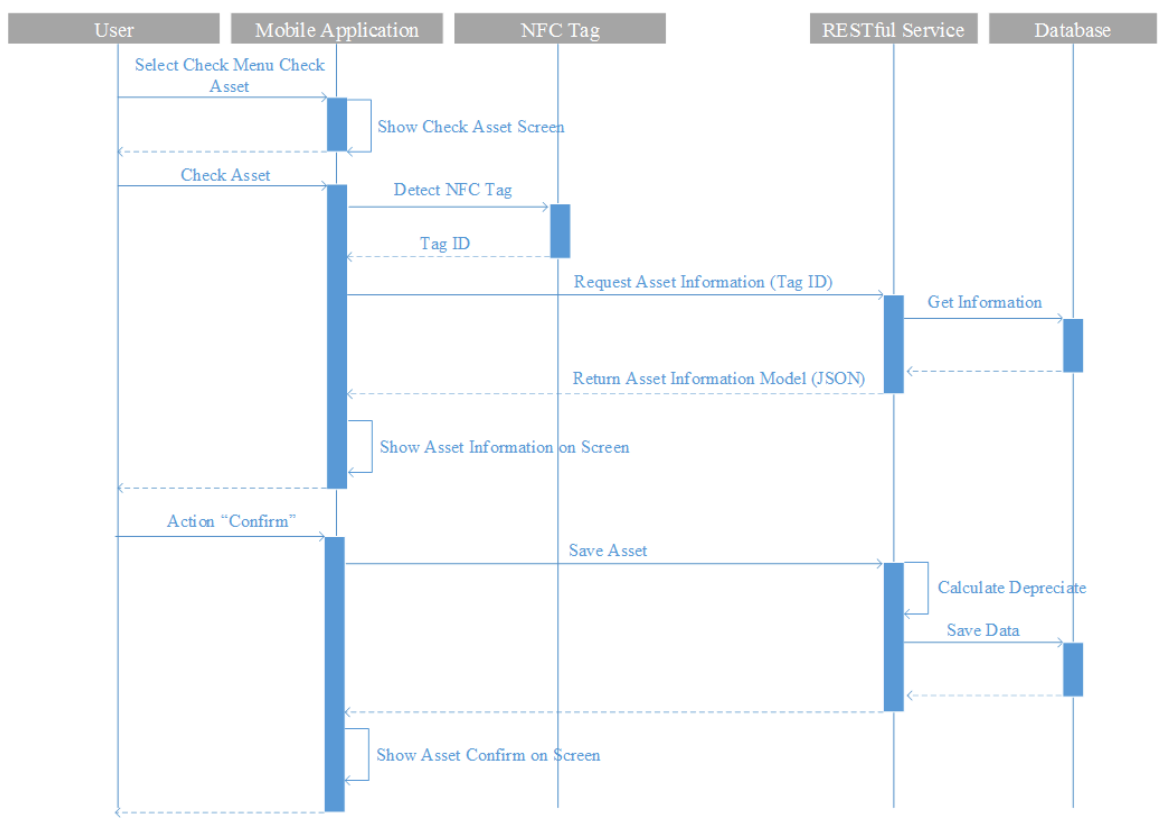

Fig. 3. Sequence Diagram: ASCMS-Based monitoring asset

In the procedures associated with monitoring assets, the user firstly selects the asset check menu from the user menu. Then, the ASCMS on a mobile can display an asset verification screen for the user. When the smartphone is found within the range of the NFC Tag, it automatically detects the NFC tag, and the ID tag is then returned to its ASCMS on the mobile to use the ID tag to request the information about the asset in the central system. Next, the central system will return the asset information model (JSON) back to the ASCMS on the mobile, and it finally displays the asset information on the smartphone screen. When the user receives data about the asset, the icon "Confirmation" is clicked. After that, the ASCMS on the mobile sends recorded data on the monitored asset to the central system, which automatically calculates the depreciation of the asset recorded in the database. Finally, the central system confirms the situation with outputs to the ASCMS on the mobile, and onto the user's monitoring screen.

\section{Mobile and Server-Side Application Architecture}

The ASCMS software architecture focuses on the overview of software structures in linkage with the correlations of sub-systems and their components. These are divided into two major parts as shown in Figure 4. 


\subsection{Part 1: Mobile application architecture}

- The User Interface (UI) functions as the Android application's screen monitor, as is used by service users. It is also shown in the form of different output and input data, supported by controlling the service users' Android application;

- Business Functions are responsible for managing the Android application's logic used for calculating the required business options;

- The Data Model functions as a data processor which is designed to access databases, files, and to stream data, as well as to convert other databases into data objects or serialization objects;

- The Communication Architecture functions as a communicator interacting with its REST service and is designed for delivering other databases using its Android application;

- The Application Utility is designed to access its library software supported for the applications of writing logs, as well as data security, and reading/writing files;

- Sensor Layer is a piece of hardware which is installed with hardware accessories to permit data accessibility and Android application, and

- The Hardware Layer is a part of the technological tags designed to access data from assets, which are confirmed for the identification and examination of the required assets.

\subsection{Part 2: Server-side application architecture}

- The Web Interface is designed to access website services particularly in terms of websites, REST services, and reporting via HTTP Protocol;

- The Data Model functions in the same way as its mobile application did;

- The Application Utility is designed for the optimum accessibility of the library software, which facilitates the applications of writing logs, ensures data security, and reading/writing files;

- Data Persistence functions as a database input or file system input that serves for storing databases in the system.

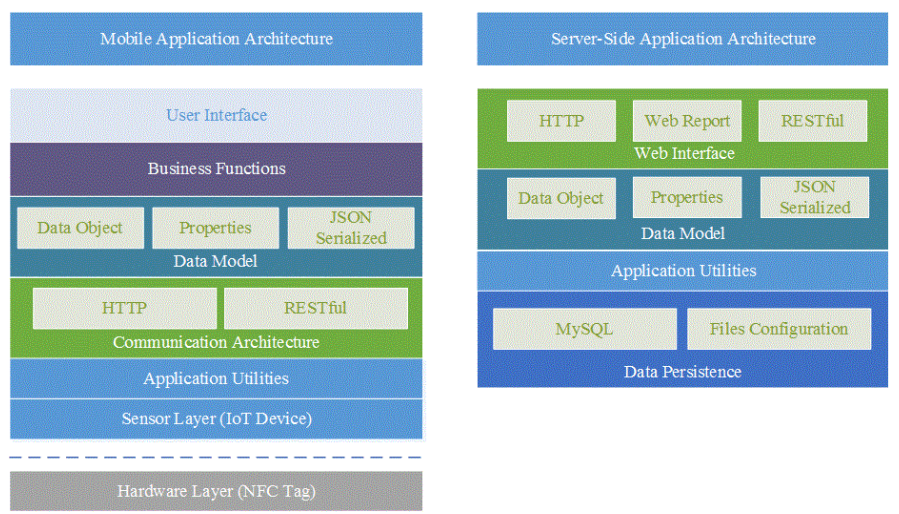

Fig. 4. Mobile and Server-Side Application Architecture of ASCMS 
The mobile application architecture, in combination with the server-side application architecture, adopts NFC tags which are placed on the devices or things that need to be assigned a serial number or a code. Within the NFC tag, the tag ID is logged to retrieve information and then to display outputs about devices such as package codes, the device's name, asset management agencies, asset stores, etc. When the smartphone with its ASCMS application is detected within the NFC range, the mobile device driver (NFC) found in the sensor layer will send the NFC ID tag displayed from the smartphone's screen. Finally, all the data mostly found in the NFC are processed in the ASCMS central system.

\section{The Asset Supply Chain Management System-based IoT Technology for HEIs}

ASCMS is used to control and monitor assets, specify the order numbers according to the Federal Stock Number (FSN), and use the NFC Tag to save and tag the asset which allows an immediate examination of the information with regard to the asset. The ASCMS application works on Android/OS. The users on the system need to pass authentication and authorization in order to access the system. The ASCMS is divided into two parts:

- The ASCMS application on the smartphone is the main application that is used to monitor and control an asset as shown in Figure 6.

- The asset information management backend is recalled by the web browser to manage the system through the Headquarter ASCMS Online Transaction Processing system (HQ ASCMS OLTP) which processes the four types of information from the database:

- Asset Transaction Processing which is divided into two parts: providing asset data and saving asset data

- Master Maintenance and Backend Operation system: This is divided into six main menus in the form of user management, asset registration, control and maintenance of assets, annual asset management, distribution, and NFC tag.

- Batch Jobs is the annual calculation of assets in the scheduled time.

- The Monitoring System or Detection System is used to alert the organization to internal defects and system malfunctionions. This makes it easier for the officers to maintain and work in the system as shown in Figure 7.

Moreover, ASCMS has a management reporting system (MRS) which is used to request information about the report to the Headquarter Data Analysis and Report. When the headquarters receives the information request, it will respond by sending the requested information through the web browser in a report and graph form. MRS can be divided into ten types of report: Report of Registered Durable Articles, Report of Asset Control Registration, Depreciation Report, Report of Usage History of Durable Articles, Report of Registered Assets, Report of Expired Durable Articles, Report of Repair History of Durable Articles, Report of Lent or Returned Assets, Annual Asset Management Report, and Registration of Durable Goods Distribution. 


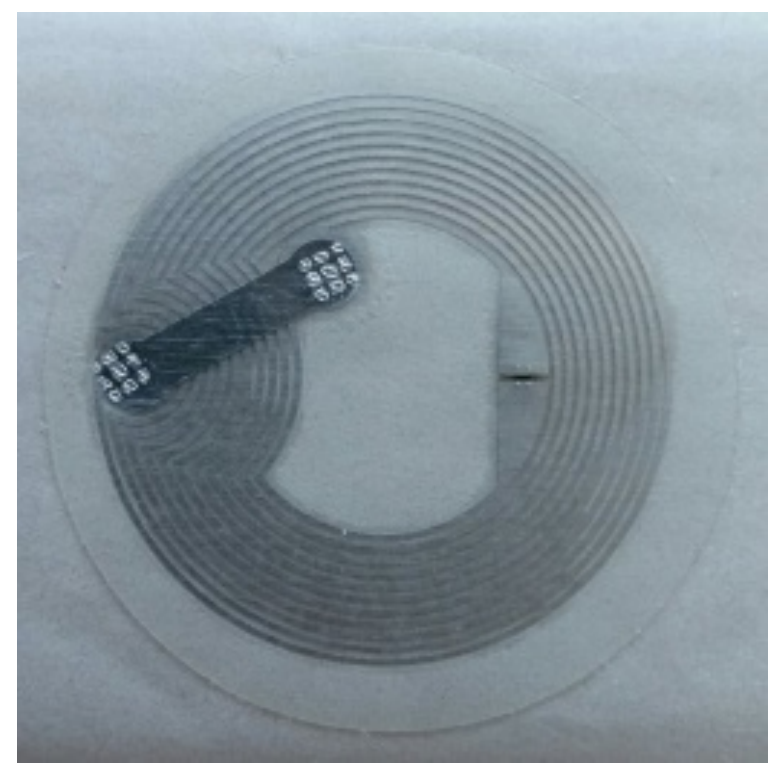

Fig. 5. NFC Tags with the NXPNTAG213 chip

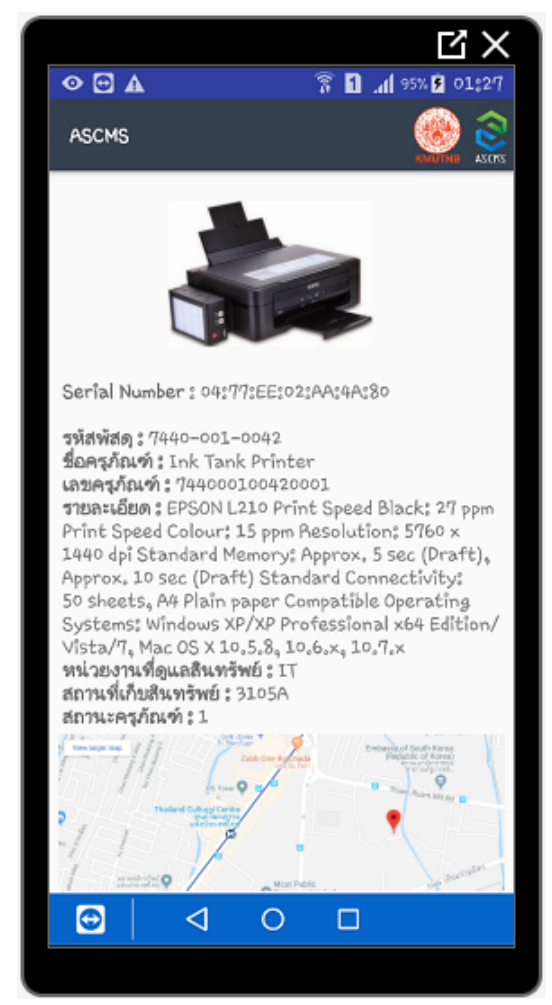

Fig. 6. The information of monitoring asset (User Interface on smartphone) 


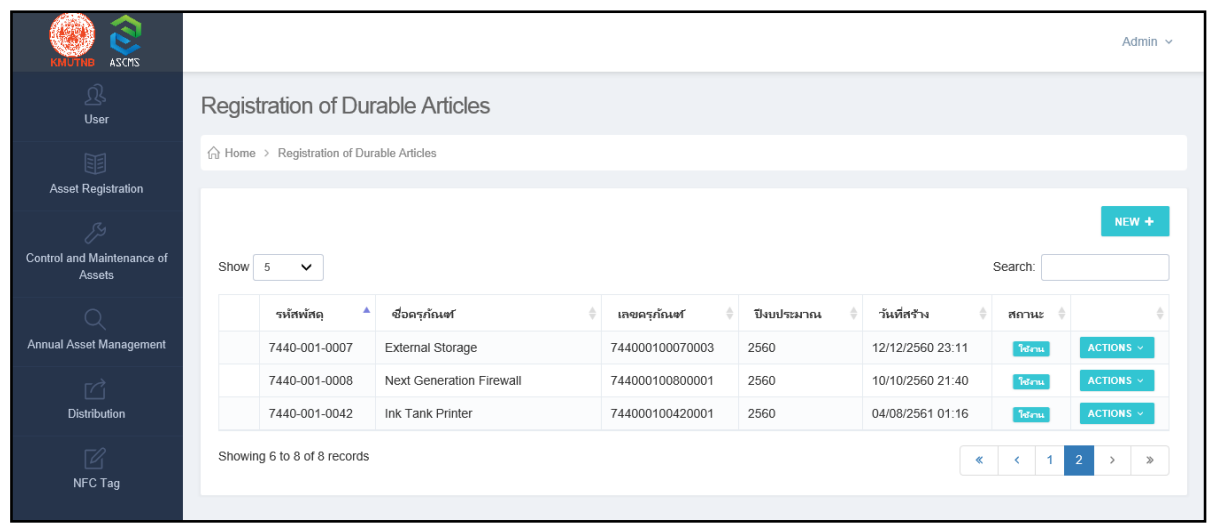

Fig. 7. The asset information management backend (User Interface on PC)

The researcher has tested the efficiency of the system with the use of black-box testing. Software Specialist, System Analyst and Software Engineer were used to test the working processes and the functions of the system, to detect any weaknesses, and to improve the system, as shown in Table 1.

Table 1. The evaluation of the performance of an asset supply chain management systembased IoT technology.

\begin{tabular}{|l|c|l|l|}
\hline \multicolumn{1}{|c|}{ Evaluation Lists } & $\overline{\boldsymbol{x}}$ & S.D. & Level of Quality \\
\hline 1. Module Test & 4.42 & 0.55 & Good \\
\hline 1.1 User & 4.45 & 0.56 & Good \\
\hline 1.2 Asset Registration & 4.52 & 0.50 & Great \\
\hline 1.3 Control and Maintenance of Assets & 4.42 & 0.50 & Good \\
\hline 1.4 Monitoring Asset Management & 4.11 & 0.47 & Good \\
\hline 1.4.1 Smartphone & 4.25 & 0.44 & Good \\
\hline 1.4.2 Backend & 3.98 & 0.46 & Good \\
\hline 1.5 Distribution & 4.39 & 0.65 & Good \\
\hline 1.6 NFC Tag & 4.61 & 0.49 & Great \\
\hline 2. System Test & 4.55 & 0.50 & Great \\
\hline 3. Usability Test & 4.52 & 0.50 & Great \\
\hline 4. Security Test & 4.58 & 0.50 & Great \\
\hline Total Average Result & 4.51 & 0.54 & Great \\
\hline
\end{tabular}

According to Table 1, the results of the evaluation indicated a great level of satisfaction with the system which indicates that it would be of value for use in Thai HEIs. The test results can be divided into four parts: the Module Test indicated a good performance level, the System Test indicated a great performance level, the Usability Test indicated a great performance level, and the Security Test indicated a great performance level. This testing procedure shows that the implemented system is in concordance with the context diagram and the graphical user interface (GUI). 


\section{Conclusion}

In this paper, the design and development of the ASCMS can be used to serve Thai higher educational institutions' internal administration and their working processes in line with real-life working situations, together with budgeting management, organizational working behaviors, and service users' requirements. All were analyzed in terms of its good governance including the rules of law, transparency and values. The concept of SCM and IoT technology can be used in terms of asset information management, in conjunction with logistics (Information Flows, Financial Flows, Asset Flows, Demand, Supply) and the processes that take place throughout the supply chain, including the impact that good governance has on the such a chain. The ASCMS is divided into 2 parts: 1) ASCMS mobile applications and 2) the backend of the ASCMS which is divided into 6 main menus: User Management, Asset Registration, Control and Maintenance of Assets, Annual Asset Management, Distribution, and NFC Tag. Users can access the information following the rights of users. Moreover, the management reporting system can represent the information which will be applied with regard to planning, monitoring, controlling and decision making in terms of the asset in the future. In this case, IoT tags can use the NFC to connect with smartphones which can be used to identify and monitor the asset. However, the ASCMS should be improved with regard to the movement of assets and real-time notification, along with the assets' locations and coordinate identification. In addition, HEIs should apply Tags Technology or others smart sensor systems to detect signal at a long distance, so that the system will be more efficient. However, this solution will increase the cost of the devices used.

\section{Acknowledgement}

The researchers would like to thank Faculty of Science and Technology, Surindra Rajabhat University, Division of Information and Communication Technology for Education, Faculty of Technical Education, Vocational Education Technology Research Center, Innovation and Technology Management Research Center at Science and Technology Research Institute, King Mongkut's University of Technology North Bangkok, which supported this research.

\section{References}

[1] An, J., Gui, X., \& He, X. (2012). Study on the Architecture and Key Technologies for Internet of Things. International Conference on Electrical and Computer Engineering. Advances in Biomedical Engineering, 11, 329-335.

[2] Anupan, A., Nilsook, P., \& Wannapiroon, P. (2015). A Framework for a Knowledge Management System in a Cloud Computing Environment Using a Knowledge Engineering Approach. International Journal of Knowledge Engineering, 146-149.

[3] Anupan, A., Nilsook, P., \& Wannapiroon, P. (2016). Knowledge Engineering Management System on Cloud Technology for Externship Students. Teaching, Assessment and 
Paper-Asset Supply Chain Management System-based IoT Technology for Higher Education Institutions

Learning for $\quad$ Engineering $\quad$ (TALE2016). IEEE, 388. https://doi.org/10.1109/TALE.2016.7851826

[4] Aparicio, J., Echevarria, J. J., \& Legarda, J. (2017). A Software Defined Networking Approach to Improve the Energy Efficiency of Mobile Wireless Sensor Networks. KSII Transactions on Internet and Information Systems, 11(6), 2848-2869. https://doi.org/1 $\underline{0.3837 / \text { tiis.2017.06.003 }}$

[5] Armenio, F., Barthel, H., Dietrich, P., Duker, J., Floerkemeier, C., Garrett, J., Harrison, M., Hogan, B., Mitsugi, J., Preishuber-Pfluegl, J., Ryaboy, O., Sarma, S., Suen, K., Traub, K., \& Williams, J. (2009). The EPCglobal Architecture Framework, EPCglobal Final Version 1.3, Approved 19 March 2009, 1-74. [Online]. Available: https://goo.gl/y3RBJo

[6] Atzori, L., Iera, A., \& Morabito, G. (2010). The Internet of Things: a survey. Computer Networks. ELSEVIER, 54(15), 2787-2805. https://doi.org/10.1016/j.comnet.2010.05.010

[7] Behrens, R., \& Ahmed, A. (2017). A Security Architecture for the Internet of Things. KSII Transactions on Internet and Information Systems, 11(12), 6092-6115, 2017. https://doi.org/10.3837/tiis.2017.12.022

[8] Benchikh, M. (2009). Data Communications and Computer Networks Chapter 4: Network Layer, King Saud University.

[9] Benyo, B., Sodor, B., Doktor, T., \& Fordos, G. (2012). Student Attendance Monitoring at the University Using NFC. Wireless Telecommunications Symposium (WTS), 1-5. https://doi.org/10.1109/WTS.2012.6266137

[10] Botterman, M. (2009). Internet of Things: An early reality of the Future Internet. Report of the Internet of Things Workshop. European Commission.

[11] Cardoso, A., Restivo, T. M., Guerra, H., \& Palma, B. L. (2017). The Use of Emerging Technologies on the Internet of Everything. International Journal of Interactive Mobile Technologies, 11(5), 4-5. https://doi.org/10.3991/ijim.v11i5.7368

[12] Chang, K., \& Chen, J. (2012). A Survey of Trust Management in WSNs, Internet of Things and Future Internet. KSII Transactions on Internet and Information Systems, 6(1), 5-23. https://doi.org/10.3837/tiis.2012.01.001

[13] Chawla, V., \& Ha, D. S. (2007). An overview of passive RFID. IEEE Communications Magazine, 45(9), 11-17. http://dx.doi.org/10.1109/MCOM.2007.4342873

[14] Chen, X., \& Jin, A. (2012). Research on Key Technology and Applications for Internet of Things. Physics Procedia, 33, 561-566.

[15] Chopra, S., \& Meindl, P. (2007). Supply Chain Management: Strategy, Planning and Operation. New Jersey, Prentice Hall, Inc.

[16] Chuachan, T., Waidee, P., \& Meesiri, S. (2017). An IoT System for Named Data Networking. The Thirteenth National Conference on Computing and Information Technology (NCCCIT2017).

[17] Darianian, M., \& Michael, M. P. (2008). RFID Master-Slave Reader Architectures for Smart Spaces Applications. Third International Conference on Pervasive Computing and Applications. IEEE, 741-746. https://doi.org/10.1109/ICPCA.2008.4783707

[18] Dobrilovec, D., Zeljko, S. (2016). Design of open-source platform for introduction Internet of Things in university curricula. International Symposium on Applied Computational Intelligence and Informatics. IEEE, 273-276. https://doi.org/10.1109/SACI.2016.7507384

[19] Dunkels, A., Vasseur, JP. (2008). IP for Smart Objects, Internet Protocol for Smart Objects (IPSO) Alliance. IPSO Alliance. White Paper \#1.

[20] Freudenthal, E., Herrera, D., Kautz, F., \& Natividad, C. (2007). Suitability of NFC for Medical Device Communication and Power Delivery. Engineering in Medicine and Biology Workshop, 51-54. https://doi.org/10.1109/EMBSW.2007.4454171. 
[21] Gershenfeld, N., Krikorian, R., \& Cohen, D. (2004). The Internet of Things. Scientific American, 291(4), 76-81.

[22] Gluhak, A., \& Presser, M. (2009). The Internet of Things Connecting the real world with the digital world. EURESCOM message.

[23] Government Gazette. (2009). Regulation of the Office of the Prime Minister to establish a system of good governance and society. [Online]. Available: http://goo.gl/US3duq

[24] Gubbi, J., Buyya, R., Marusic, S., \& Palaniswami, M. (2013). Internet of Things (IoT): A vision, architectural elements, and future directions. Future Generation Computer Systems. ELSEVIER, 29(7), 1645-1660. https://doi.org/10.1016/j.future.2013.01.010

[25] Guinard, D. (2011). A Web of Things Application Architecture - Integrating the RealWorld into the Web. ETH Zurich.

[26] Guinard, D., Trifa, V. (2009). Towards the Web of Things: Web Mashups for Embedded Devices. WWW (International World Wide Web Conferences), Enterprise Mashups and Lightweight Composition on the Web (MEM 2009) Workshop.

[27] Guinard, D., Trifa, V. (2015). Building the Web of Things. Manning.

[28] Guinard, D., Trifa, V., \& Wilde, E. (2010). A Resource Oriented Architecture for the Web of Things. Internet of Things 2010 International Conference (IoT 2010). https://doi.org/10.1109/IOT.2010.5678452

[29] Guinard, D., Trifa, V., Mattern, F. \& Wilde, E. (2011). 5 From the Internet of Things to the Web of Things: Resource Oriented Architecture and Best Practices. Springer. 97-129.

[30] Guinard, D., Trifa, V., Pham, T., \& Liechti, O. (2009). Towards physical mashups in the Web of Things. 1-8. https://doi.org/10.1109/INSS.2009.5409925.

[31] Haykin, S. (2005). Cognitive radio: brain-empowered wireless communications. IEEE Journal on Selected Areas in Communications, 23(2), 201-220. https://doi.org/1 0.1109/JSAC.2004.839380

[32] Hui, J., Culler, D., Chakrabarti, S. (2009). 6LoWPAN: Incorporating IEEE802.15.4 Into the IP Architecture - Internet Protocol for Smart Objects (IPSO) Alliance, IPSO Alliance. White Paper \#3.

[33] Husni, E. (2017). Driving and Fuel Consumption Monitoring with Internet of Things. International Journal of Interactive Mobile Technologies, 11(3), 78-97.

[34] Information Sciences Institute. (1981) Internet Protocol DARPA Internet Program Protocol Specification. University of Southern California. Marina del Rey, California.

[35] INFSO D.4 Networked Enterprise \& RFID INFSO G.2 Micro \& Nanosystems in: cooperation with the RFID working group of the EPOSS. Internet of Things in 2020, A Roadmap for the future. European Commission.

[36] International Telecommunication Union. (2005). The Internet of Things. ITU Internet Reports.

[37] Juels, A. (2006). RFID security and privacy: a research survey. IEEE Journal on Selected Areas in Communications, 24(2), 381-394.

[38] Khare, R., \& Celik, T. (2006). Microformats: A pragmatic path to the semantic web. Proceedings of the 15th International World Wide Web Conference (WWW2006), 865-866.

[39] Kuandee, W., Nilsook, P., \& Wannapiroon, P. (2018). Supply Chain Asset Management Process with the Internet of Things for Good Governance for Higher Education Institutions. International Journal of the Computer, the Internet and Management (IJCIM), 26(2).

[40] Luo, S., Cheng, L., \& Ren, B. (2014). Practical Swarm Optimization based FaultTolerance Algorithm for the Internet of Things. KSII Transactions on Internet and Information Systems, 8(4), 1178-1191. https://doi.org/10.3837/tiis.2014.04.001 
[41] Madakam, S., Ramaswamy, R., \& Tripathi, S. (2015). Internet of Things (IoT): A Literature Review. Journal of Computer and Communications, 3, 164-173. http://dx.doi.org/10.4236/jcc.2015.35021

[42] Mentzer, J. T., DeWitt, W., Keebler, J. S., Min, S., Nix, N. W., Smith, C. D. \& Zacharia, Z. G. (2001). What is supply chain management? In Mentzer, J.T. (Ed.), Supply Chain Management, Sage, Thousand Oaks, CA, 1-25.

[43] Misra, G., Kumar, V., Agarwal, A., \&Agarwal, K. (2016). Internet of Things (IoT) - A Technological Analysis and Survey on Vision, Concepts, Challenges, Innovation Directions, Technologies, and Applications (An Upcoming or Future Generation Computer Communication System Technology). American Journal of Electrical and Electronic Engineering, 4(1), 23-32. http://dx.doi.org/10.12691/ajeee-4-1-4

[44] Nagurney, A. (2006). Supply Chain Network Economics: Dynamics of Prices, Flows, and Profits, Edward Elgar Publishing.

[45] Near Field Communication Organization. (2012). About Near Field Communication. [Online]. Available: https://goo.gl/H7BpmQ

[46] Near Field Communication Organization. (2012). Near Field Communication versus Bluetooth. [Online]. Available: https://goo.gl/KtS86K

[47] Nookhong, J., \& Nilsook, P. (2016). System Architecture for Green University Resource Planning on Cloud Computing. International Journal of the Computer, the Internet and Management, 24(2), 1-7.

[48] Nookhong, J., \& Nilsook, P. (2017). Green University Resource Planning on Cloud Computing. International Journal of Information and Education Technology, 7(10), 722-727.

[49] Nosowitz, D. (2011). Everything You Need to Know About Near Field Communication. Popular Science Magazine, [Online]. Available: https://goo.gl/9jYjYh

[50] Office of the National Economic and Social Development Board. (2013). Strategic Plan for the development of logistics of Thailand Vol.2 (2013-2017). [Online]. Available: http://goo.gl/2mAlVL.

[51] Piyare, R., Lee, S. R. (2013). Towards Internet of Things (IoTs): Integration of wireless sensor network to Cloud services for data collection and SharingII, International Journal of Computer Networks \& Communications (IJCNC), 5(5), 59-72. https://doi.org/10.51 21/ijenc.2013.5505

[52] Poovarawan, Y. (2016). Internet of Things on Cloud and Big Data for Thailand 4.0. Mahidol Libraries Annual Conference 2016. [Online]. Available: https://goo.gl/KCpb9U

[53] Prime Minister's Office. (2009). Regulations of the Office of the Prime Minister on Procurement B.E.2535 (1992). [Online]. Available: https://goo.gl/7rXcR2

[54] Razzak, F. (2012). Spamming the Internet of Things: A Possibility and its probable Solution. The 9th International Conference on Mobile Web Information Systems. Procedia Computer Science, 10, 658-665. http://dx.doi.org/10.1016/j.procs.2012.06.084

[55] Saraubon, K., Nilsook, P., \& Wannapiroon, P. (2016). System Design of Mobile Augmented Book. International Journal of Interactive Mobile Technologies (iJIM), 10(1), 5259. http://dx.doi.org/10.3991/ijim.v10i1.5276

[56] Sundmaeker, H., Guillemin, P., Friess, P., \& Woelffle, S. (2010). Vision and challenges for Realising the Internet of Things. Cluster of European Research Projects on the Internet of Things (CERP IoT). European Commission.

[57] Trifa, M. V. (2010). Building Blocks for a Participatory Web of Things: Devices, Infrastructures, and Programming Frameworks. ETH Zurich.

[58] Ungurean, I., Gaitan C. N., \& Gaitan, G. V. (2016). A Middleware Based Architecture for the Industrial Internet of Things. KSII Transactions on Internet and Information Systems, 10(7), 2874-2891. http://dx.doi.org/10.3837/tiis.2016.07.001 
[59] Yuan, R., Shumin, L., \& Baogang, Y. (2007). Value Chain Oriented RFID System Framework and Enterprise Application, Science Press, Beijing.

\section{$9 \quad$ Authors}

Watjanarat Kuandee is with the Faculty of Science and Technology, Surindra Rajabhat University, Surin, Thailand. (e-mail: watjanarat.k@srru.ac.th)

Prachyanun Nilsook works as associate professor at Faculty of Technical Education and also the Head of Vocational Educational Technology Research Center, King Mongkut's University of Technology North Bangkok, Bangkok, Thailand. (e-mail: prachyanunn@kmutnb.ac.th)

Panita Wannapiroon works as associate professor at Division of Information and Communication Technology for Education, and Director of Innovation and Technology Management Research Center, Science and Technology Research Institute, King Mongkut's University of Technology North Bangkok, Bangkok, Thailand. (e-mail: panita.w@fte.kmutnb.ac.th)

Article submitted 05 March 208. Resubmitted 21 August and 21 November 2018. Final acceptance 15 January 2019. Final version published as submitted by the authors. 